\title{
Irreversible nucleation in molecular beam epitaxy: From theory to experiments
}

\author{
Paolo Politi ${ }^{1,2}$, 周 and Claudio Castellano ${ }^{3}$ 用 \\ ${ }^{1}$ Istituto di Fisica Applicata "Nello Carrara", Consiglio Nazionale delle Ricerche, Via Panciatichi 56/30, 50127 Firenze, Italy \\ ${ }^{2}$ Istituto Nazionale per la Fisica della Materia, Unità di Firenze, \\ Via G. Sansone 1, 50019 Sesto Fiorentino, Italy \\ ${ }^{3}$ Dipartimento di Fisica, Università di Roma "La Sapienza", \\ and Istituto Nazionale per la Fisica della Materia, \\ Unità di Roma 1, P.le A. Moro 2, I-00185 Roma, Italy
}

(Dated: November 10, 2018)

\begin{abstract}
Recently, the nucleation rate on top of a terrace during the irreversible growth of a crystal surface by MBE has been determined exactly. In this paper we go beyond the standard model usually employed to study the nucleation process, and we analyze the qualitative and quantitative consequences of two important additional physical ingredients: the nonuniformity of the Ehrlich-Schwoebel barrier at the step-edge, because of the existence of kinks, and the steering effects, due to the interaction between the atoms of the flux and the substrate. We apply our results to typical experiments of second layer nucleation.
\end{abstract}

PACS numbers: 81.10.Aj, 68.55.Ac, 68.55.-a

\section{INTRODUCTION}

Nucleation is a key process during the growth of a crystal by Molecular Beam Epitaxy (MBE), when the substrate is oriented along a high symmetry direction. In that case, freshly deposited atoms diffuse on the surface until they meet other adatoms (nucleation process) or the step of a growing terrace (aggregation process). Layers are completed through island coalescence and the filling of vacancies.

Nucleation is irreversible if a dimer (i.e., a nucleus formed by the meeting of two adatoms) is thermally stable. This condition depends on the two most important external parameters: the temperature $T$ and the intensity $F$ of the flux. It holds if the dissociation time of a dimer (which grows with decreasing $T$ ) is larger than the time (decreasing with $F$ ) required to aggregate more adatoms to the dimer and to stabilize it.

As a matter of fact, nucleation appears to be irreversible, in a range of temperatures experimentally ac cessible, for several experimental systems as $\mathrm{Pt} / \mathrm{Pt}(111), \mathrm{t}$ $\mathrm{Ag} / \mathrm{Ag}(100)$, $\mathrm{E} \mathrm{Fe} / \mathrm{Fe}(100)$, 自 and $\mathrm{Ag} / \mathrm{Ag}(111)$.

Except for the submonolayer regime, where adatoms diffuse on the substrate, nucleations occur on terraces, and most of them take place on 'top terraces', 5 which are defined as those surrounded by a single closed descending step.

In some recent paper 5 .6 we have studied the nucleation process on top of a terrace: we have evaluated the number $\omega$ of nucleation events per unit time (nucleation rate) and their spatial distribution. The total nucleation rate $\omega$ is of great importance in several respects: it determines the typical distance between nucleation centers in the submonolayer regime, the so-called diffusion length $\ell_{\mathrm{D}}$ (see Ref. 1); it allows to extract the additional energy barrier for interlayer diffusion [Ehrlich-Schwoebel (ES) barrier] from an experiment of second layer nucleation; it is the main factor determining the stable or unstable character of growth during its first stages

In Sec. II of this paper we report the formula giving the exact rafue of the nucleation rate $\omega$ that was derived previously 6 .6 Such a formula depends on the quantity $W$, the probability that two adatoms deposited simultaneously actually meet. Here we evaluate $W$ explicitly for all values of the terrace size $L$ and of the so-called ES length, $\ell_{\mathrm{ES}}=\frac{\nu}{\nu^{\prime}}-1$, which measures the difference between the intra-layer hopping rate $\nu$ and the inter-layer hopping rate $\nu^{\prime}$. The resulting formula is extremely accurate. In this way $\omega$ is written in full generality in terms only of $L, F, \nu, \nu^{\prime}$ and some geometrical constants that are given explicitly for the most relevant cases. We also provide the approximate formulas which are correct in some limiting regimes.

Later we discuss, both from a qualitative and a quantitative point of view, two issues which are believed to be important experimentally and that are not included in the 'standard' models used for studying nucleation: the effects of nonuniform interlayer barriers and of steering phenomena in the deposition flux.

The 'standard' model for nucleation assumes the presence of an interlayer barrier which is uniform along the step surrounding the top terrace. However, even for compact terraces, kinks are unavoidably present along the step and they are preferential sites for descending 0 because the ES barrier is expected to be reduced by the increased number of neighbours. The 'effective' barrier $\Delta E_{\text {eff }}$ is therefore a combination of the barrier $\Delta E_{k}$ at kinks and the higher barrier $\Delta E_{0}$, felt by an adatom along straight steps. Even more importantly, since the number of kinks is temperature and coverage dependent, $\Delta E_{\text {eff }}$ depends on $T$ and coverage, even if $\Delta E_{k}$ and $\Delta E_{0}$ do not. This issue is treated in Sec. III.

The 'standard' model also assumes a uniform flux $F$ impinging on the terrace. However, it has been shown10 11.12 that the attractive interaction between the incoming atom and the atoms incorporated in the grow- 
ing surface increases the number of particles landing on a top terrace. This may clearly affect the nucleation rate. The relevance of this effect strongly depends on the kinetic energy of the incoming particles, the strength of the interaction and the angle of deposition. A minimal model, where all these factors determine a single adimensional parameter $\epsilon$, is discussed in Sec. IV.

In Sec. V we apply our results to typical experiments of second layer nucleation: an experiment on $\mathrm{Pt} / \mathrm{Pt}(111)$ at different CO partial pressures, 13 and an experiment on $\mathrm{Ag} / \mathrm{Ag}(111)$ at different temperatures 1 In this way we are able to discuss the relevance of nonuniform barriers and steering effects in two real cases.

Concluding remarks are presented in Sec. VI.

\section{THE NUCLEATION RATE}

In Refs. 5 and 6 we have evaluated exactly the nucleation rate $\omega$ for a terrace of linear size $L$. This quantity is adimensional and represents the number of atoms along the edge, for a polygon-shaped island, or along the radius, for a circular island. The total number of atoms in the island is $\mathcal{A}=\gamma L^{2}$. The most general expression for the nucleation rate is:

$$
\omega=\mathcal{F} \frac{\tau_{\text {res }}}{\tau_{\text {res }}+\tau_{\text {dep }}} W,
$$

where $\mathcal{F}$ is the number of atoms arriving on the terrace per unit time, $\tau_{d e p}$ and $\tau_{\text {res }}$ are the deposition and residence time, respectively, and $W$ is the probability that two adatoms, deposited simultaneously, meet before leaving the terrace.

For a uniform flux, $\mathcal{F}=F \mathcal{A}$, and its inverse is the average time between deposition events, the deposition time, $\tau_{\text {dep }}=1 / \mathcal{F}$. The residence time $\tau_{\text {res }}$ is the average time spent by a single particle on the terrace before descending from it. It depends on the size $L$ of the terrace and on the strength of the additional ES barrier, which can be quantified via the (adimensional) ES length, $\ell_{\mathrm{ES}}=$ $\frac{\nu}{\nu^{\prime}}-1$. The rates $\nu, \nu^{\prime}$ for intra and inter-layer hoppingwhose precise definition is given in App. A- are generally written as a prefactor times an Arrhenius factor, $\nu=\nu_{0} \exp \left(-E_{d} / k_{\mathrm{B}} T\right), \nu^{\prime}=\nu_{0}^{\prime} \exp \left[-\left(E_{d}+\Delta E\right) / k_{\mathrm{B}} T\right]$. In the simple case where $\nu_{0}$ and $\nu_{0}^{\prime}$ are supposed the same, the ES length is $14 \ell_{\mathrm{ES}}=\exp \left(\Delta E / k_{\mathrm{B}} T\right)-1$ In terms of $L$ and $\ell_{\mathrm{ES}}$, the residence time is given by 15

$$
\tau_{\text {res }}=\left(\alpha \ell_{\mathrm{ES}}+\beta L\right) L / \nu,
$$

which allows to distinguish in an easy way the 'weak barrier' regime $\left(\ell_{\mathrm{ES}} \ll L, \tau_{\text {res }}=\beta L^{2} / \nu\right)$ from the 'strong barrier' one $\left(\ell_{\mathrm{ES}} \gg L, \tau_{\text {res }}=\alpha L \ell_{\mathrm{ES}} / \nu=\alpha L / \nu^{\prime}\right)$. The numerical factors $\alpha$ and $\beta$, as well as $\gamma$, depend on the shape of the terrace and on the symmetry of the underlying lattice. Their values for some relevant cases have been determined numerically or analytically and are reported in Table I. In App. B we determine analytically the geometrical parameters relevant for a circular terrace.
It is worth noting that the parameter $\alpha$ can easily be written in terms of $\gamma$ and other geometrical factors. As a matter of fact, in the limit of large ES barriers, the residence time has two equivalent expressions. The first comes from Eq. (2), $\tau_{\text {res }}=\alpha L / \nu^{\prime}$. The second is found by considering that the escape rate from the terrace, $\tau_{\text {res }}^{-1}$, is given by the probability of finding an adatom on an edge site, times the fraction of hops leading from an edge site to the lower terrace, times the interlayer hopping rate. The fraction of jumps leading to the lower terrace is equal to $\Delta z / z$, where $z$ is the coordination number of the lattice, and $\Delta z$ is the number of missing neighbours for an edge site. In the strong barrier regime the probability distribution of adatoms on the terrace is uniform and hence the probability of finding an adatom on an edge site is $\mathcal{P} / \mathcal{A}$, where $\mathcal{P}=\eta L$ is the perimeter of the terrace in units of lattice sites. Summing up, we can write

$$
\tau_{\text {res }}=\frac{\mathcal{A}}{\mathcal{P}} \frac{z}{\Delta z} \frac{1}{\nu^{\prime}}=\frac{\gamma z}{\eta \Delta z} \frac{L}{\nu^{\prime}}, \quad\left[\ell_{\mathrm{ES}} \gg L\right]
$$

so that $\alpha=\gamma z /(\eta \Delta z)$. The quantities $\eta$ and $\Delta z$ are reported in Table (see also App. B).

The quantity $W$ in Eq. (11), the probability that two atoms both on the terrace actually meet, depends on the initial spatial distributions for the two atoms, but this dependence is very weak and can in practice be neglected. 16 The probability $W$ does not change much even if one atom is taken as immobile.5 In such a case, $W$ can easily be written in terms of properties of a single random walk

$$
W=\frac{N_{\mathrm{dis}}\left(L, \ell_{\mathrm{ES}}\right)}{\mathcal{A}},
$$

where $N_{\mathrm{dis}}\left(L, \ell_{\mathrm{ES}}\right)$ is the number of distinct sites visited by a single particle before leaving the terrace and clearly depends on $\ell_{\mathrm{ES}}$ and $L$. For extremely strong barriers, the atom is able to visit all sites, $N_{\text {dis }} \rightarrow \mathcal{A}$, so that $W \rightarrow 1$ In the opposite limit of very weak barriers, it is known 17 that $N_{\text {dis }}(L, 0)=N_{0} L^{2} / \ln \left(L / L_{0}\right)$ (so that $W \approx 1 / \ln L$ ). An approximate formula interpolating between the two limits is given in Ref. 5. Here we improve on that estimate by providing an analytic expression that reproduces with very good accuracy the value of $W$ obtained by numerical simulations for all values of $\ell_{\mathrm{ES}}$. The formula is

$$
W=\frac{N_{1} \ell_{\mathrm{ES}}+\frac{N_{0} L \ln \left(L / L_{1}\right)}{\ln \left(L / L_{0}\right)}}{N_{1} \ell_{\mathrm{ES}}+\gamma L \ln \left(L / L_{1}\right)} .
$$

The values of $N_{0}, L_{0}, N_{1}, L_{1}$ depend on the shape of the terrace and on the symmetry of the underlying lattice, as $\alpha, \beta$ and $\gamma$ do. Their values are given for relevant terrace shapes and lattice types in Table 1 . The derivation of Eq. (5) is given in App. $\mathrm{Q}$ and its accuracy is demonstrated in Fig. 1. Notice that no parameter is fitted.

By inserting Eq. (2) and Eq. (5) into Eq. (1) we obtain the general fully explicit formula for the nucleation rate. Eq. (11) is based on the sole hypothesis that the 
TABLE I: Numerical values of the parameters $\alpha$ and $\beta$ [appearing in $\tau_{\text {res }}$, Eq. (2)], $\gamma$ (appearing in $\mathcal{A}$ ), $\eta$ (appearing in

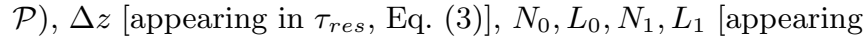
in $W$, Eq. (5)]. They are given for different shapes of the terrace and for two types of lattice: the square and the triangular one. They are typical of the (100) and (111) face of a cubic lattice, respectively. For triangular $(\triangle)$, square $(\square)$, and hexagonal $(\square)$ islands, $L$ is the edge of the polygon. For circular $(\bigcirc)$ terrace, $L$ is the radius. The values of $\alpha$ are computed using the formula $\alpha=\gamma z /(\eta \Delta z)$ [see Eq. (3)] and agree with numerical results. The values of $N_{0}, L_{0}, N_{1}$ and $L_{1}$ are determined by fitting numerical results for $N_{\text {dis }}(L)$ and $N_{\mathrm{dis}}^{\mathrm{BB}}(L)$ to their analytical expressions.

\begin{tabular}{|c|c|c|c|c|c|c|c|c|c|}
\hline & $\alpha$ & $\beta$ & $\gamma$ & $\eta$ & $\Delta z$ & $N_{0}$ & $L_{0}$ & $N_{1}$ & $L_{1}$ \\
\hline \multirow{2}{*}{$\begin{array}{r}(100) \square \\
\square\end{array}$} & 1 & 0.14 & 1 & 4 & 1 & 0.2 & 1.0 & 1.8 & 0.5 \\
\hline & $\pi / 2$ & 0.5 & $\pi$ & $4 \sqrt{2}$ & $\sqrt{2}$ & 0.7 & 0.5 & 3.1 & 0.3 \\
\hline \multirow[t]{3}{*}{$\triangle$} & $1 / 2$ & 0.05 & $1 / 2$ & 3 & 2 & 0.08 & 2.3 & 0.9 & 1.6 \\
\hline & $3 / 2$ & 0.4 & 3 & 6 & 2 & 0.7 & 0.5 & 3.0 & 0.4 \\
\hline & $\pi / 2$ & 0.5 & $2 \pi / \sqrt{3}$ & $4 \sqrt{3}$ & 2 & 0.7 & 1.0 & 3.8 & 0.2 \\
\hline
\end{tabular}

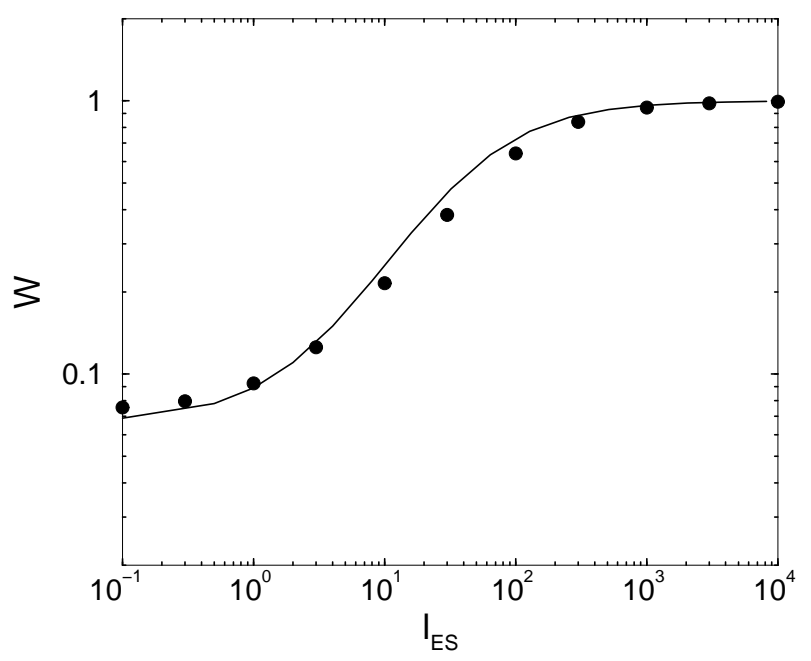

FIG. 1: Comparison of the exact numerical values of $W$ (points, see Ref. 月), with the approximate analytic formula (line) given in Eq. (5), for a square lattice and a square terrace of size $L=20$.

deposition time $\tau_{\text {dep }}$ is much larger than the so-called 'traversal time' $\tau_{t r}=\beta L^{2} / \nu$, defined as the average time an atom needs to reach the edge of the terrace (note that $\tau_{t r}=\tau_{r e s}$, in the regime $\left.\ell_{\mathrm{ES}} \ll L\right)$. . The condition $\tau_{\text {dep }} \gg \tau_{t r}$ is equivalent 5 to the condition $\ell_{\mathrm{D}} \gg 1$. A quantitative evaluation for the experimental systems discussed in Sec. $\mathrm{V}$ gives in all cases $\tau_{t r} / \tau_{d e p}<10^{-5}$.

In most cases, also the residence time is much smaller than the deposition time. For example, for the experimental systems studied in Sec. $\mathrm{V}$ we have $\tau_{\text {res }} /\left.\tau_{\text {dep }}\right|_{\mathrm{Pt}}<$ $5 \times 10^{-3}$ and $\tau_{\text {res }} /\left.\tau_{\text {dep }}\right|_{\mathrm{Ag}}<10^{-2}$. If the condition $\tau_{\text {res }} \ll \tau_{\text {dep }}$ is satisfied, the nucleation rate is just given by the expression:

$$
\begin{aligned}
\omega & =\frac{\tau_{r e s}}{\tau_{d e p}^{2}} \cdot W \quad\left[\tau_{r e s} \ll \tau_{d e p}\right] \\
& =\gamma^{2} \frac{F^{2}}{\nu} L^{5}\left(\alpha \ell_{\mathrm{ES}}+\beta L\right) \cdot W,
\end{aligned}
$$

where $W$ is given in Eq. (5). In the limit of strong barriers, if we approximate $W$ by one, we have the simpler expression: 18

$$
\omega=\alpha \gamma^{2} \frac{F^{2} L^{5}}{\nu^{\prime}} . \quad\left[\tau_{\text {res }} \ll \tau_{\text {dep }} \text { and } \frac{\nu}{\nu^{\prime}} \gg L\right]
$$

\section{THE EFFECT OF NON UNIFORM BARRIERS}

An adatom leaves the terrace because it reaches an edge site and it jumps down with a hopping rate $\nu^{\prime}$ : in the usual model for nucleation, $\nu^{\prime}$ is supposed to be the same along the step edge. However, its value may actually be non uniform: a growing step always has some degree of roughness, which makes the descent preferable from certain edge sites, and toward certain directions. For ap fcc(111) surface, the picture is even more complicated, because steps can be of two different types (A-step and B-step) and they can both be present around the same terrace. In this case their barriers are different even if both types of steps are straight. Therefore, if the standard model is adopted, the interlayer hopping rate should be understood as an effective one, valid at well defined temperature and growth conditions. Any factor able to affect the step morphology may affect $\nu^{\prime}$ as well. The 'effective' character of $\nu^{\prime}$ makes its separation in a prefactor and an exponential factor arbitrary. For this reason we are going to assume $\nu_{0}^{\prime}=\nu_{0}$.

The case of a generic distribution of ES barriers can not be treated analytically, because it is not possible to find the general expressions for $\tau_{\text {res }}$ and $W$. In the following we are assuming to be in the 'strong barrier' regime, where $W=1$ and an analytical derivation of $\tau_{\text {res }}$ is indeed possible. In this regime, the adatom density is uniform and Eq. (3) is easily generalized to non-uniform barriers:

$$
\tau_{\text {res }}=\frac{\mathcal{A}}{\mathcal{P}} \frac{z}{\langle\Delta z\rangle} \frac{1}{\left\langle\nu^{\prime}\right\rangle} .
$$

Here $\langle\Delta z\rangle$ is the number of directions leading to a hop downwards (averaged over edge sites), and $\left\langle\nu^{\prime}\right\rangle$ is the average hopping rate.

Let us consider a simplified 'kink-model': the ES barrier is everywhere equal to the high value $\Delta E_{0}$, except for special sites (and paths), where the additional barrier takes the smaller value $\Delta E_{k}$. In other words, the distribution is bimodal

$$
\Delta E= \begin{cases}\Delta E_{k} & \text { with probability } c_{k} \\ \Delta E_{0} & \text { with probability }\left(1-c_{k}\right) .\end{cases}
$$


Eq. (9) is valid as long as even the smallest of the barriers is sufficiently large to ensure a uniform probability of finding the adatom on the terrace. Hence the criterion for its validity is that the smallest ES length is much larger than the linear size of the terrace,

$$
\left(\ell_{\mathrm{ES}}\right)_{k} \equiv \mathrm{e}^{-\Delta E_{k} / k_{\mathrm{B}} T}-1 \gg L .
$$

Provided this is true, the effective barrier is determined by the relation

$$
\mathrm{e}^{-\Delta E_{\text {eff }} / k_{\mathrm{B}} T}=c_{k} \mathrm{e}^{-\Delta E_{k} / k_{\mathrm{B}} T}+\left(1-c_{k}\right) \mathrm{e}^{-\Delta E_{0} / k_{\mathrm{B}} T},
$$

whose general solution is

$$
\begin{aligned}
& \Delta E_{\text {eff }}=\Delta E_{k}+k_{\mathrm{B}} T \ln \left(\frac{1}{c_{k}}\right) \\
& -k_{\mathrm{B}} T \ln \left[1+\left(\frac{1}{c_{k}}-1\right) \exp \left[-\left(\Delta E_{0}-\Delta E_{k}\right) / k_{\mathrm{B}} T\right]\right] .
\end{aligned}
$$

In the case when the two barriers are practically the same, Eq. (13) obviously gives $\Delta E_{\text {eff }} \approx \Delta E_{k} \approx \Delta E_{0}$. In the more interesting opposite limit, when $\Delta E_{0}-\Delta E_{k}$ is larger than $k_{\mathrm{B}} T \ln \left(1 / c_{k}-1\right)$, the last term on the righthand-side is negligible, and the effective barrier has the simplified expression,

$$
\Delta E_{\text {eff }}=\Delta E_{k}+k_{\mathrm{B}} T \ln \left(\frac{1}{c_{k}}\right) .
$$

Eq. (14) has a transparent physical meaning: adatoms leave the terrace at kink sites only, but they feel an effective barrier larger than $\Delta E_{k}$, because of the finite concentration of kinks. If $T=300 \mathrm{~K}$ and $c_{k}=0.1$, the barrier increase due to this effect is $0.06 \mathrm{eV}$.

What happens when the condition (11) does not hold because $\Delta E_{k}$ is not large enough? In that case the probability to find the adatom at a kink site is suppressed and the use of Eqs. (9) and (14) underestimates $\tau_{\text {res }}$. This is clearly shown in Fig. 2 where we compare, for a square terrace on a square lattice, numerical results (symbols) for $\tau_{\text {res }}$ (in units $1 / \nu=1$ ) with the analytical approximation (lines) derived from Eqs. (9) and (14). In the case $\Delta E_{0}=\infty$ and $\Delta E_{k}=0$ the analytical formula gives a residence time (dashed line) that is well below the numerical results (diamonds). In the opposite case of $\Delta E_{0}=\infty$ and $\left(\ell_{\mathrm{ES}}\right)_{k}=\exp \left(\Delta E_{k} / k_{\mathrm{B}} T\right)-1=99$, the condition (11) is fulfilled: numerical (circles) and analytical results (solid line) agree.

Eq. (14) clearly applies not only to the case of kinks, but holds whenever there are two energy barriers for interlayer transport, one of which is significantly larger: For example for an hexagonal terrace on a (111) surface, surrounded by three A-steps and three B-steps. If $\Delta E_{A, B}$ are the ES barriers at the two different steps, the effective barrier is equal to the smaller one plus a small correction term.19

$$
\Delta E_{\text {eff }}=\min \left\{\Delta E_{A}, \Delta E_{B}\right\}+k_{\mathrm{B}} T \ln 2 .
$$

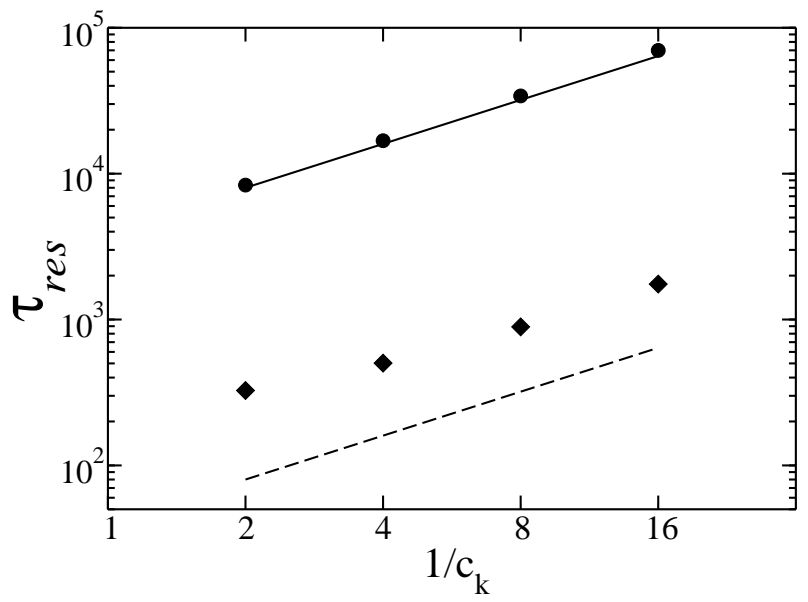

FIG. 2: The residence time (in units $1 / \nu=1$ ) for an adatom on a square terrace of size $L=40$, as a function of the inverse of the kink concentration (log-log scale). $\Delta E_{0}=\infty$ in all cases, while $\Delta E_{k}$ corresponds to $\left(\ell_{\mathrm{ES}}\right)_{k}=0$ for diamonds and to $\left(\ell_{\mathrm{ES}}\right)_{k}=99$ for circles. Symbols refer to the exact numerical calculation of $\tau_{\text {res }}$ and lines to the analytical approximation, Eqs. (9,14).

\section{STEERING EFFECTS}

The flux of incoming atoms is usually supposed to be uniform. This hypothesis is correct down to a distance of a few nanometers from the surface, but it breaks down at smaller distances because of the adatom-substrate interaction. 10 This interaction is attractive, so that it increases the effective flux landing on a top terrace.11 12

The effect is illustrated in Fig. 3 for normal deposition on a circular terrace. Because of the attractive interaction with the terrace, the deposited atom may deviate from its rectilinear trajectory $t_{1}$ and land on the terrace. The incoming flux $\mathcal{F}$, equal to $F \mathcal{A}=F \gamma L^{2}$ in the absence of steering, is therefore increased to the larger value $\mathcal{F}=F \mathcal{A}_{\text {eff }}$, since the effective capture area of the terrace is larger. The additional area for large $L$ is simply $d \mathcal{P}$, where the quantity $d$ denotes the in-plane displacement of a particle landing on the terrace edge (trajectory $t_{2}$ ) and $\mathcal{P}$ is the island perimeter. This leads to the effective flux

$$
\mathcal{F}=F(\mathcal{A}+d \mathcal{P})=F\left(\gamma L^{2}+\epsilon L\right),
$$

where $\epsilon=\eta d$ is an adimensional quantity. The actual value of $d$ depends on the thermal energy of the incoming particles and on the interaction energy between the atom and the substrate. For oblique incidence, it also depends on the angle of incidence and it should be meant as an average over the island perimeter.

The effect of the parameter $\epsilon$ will be illustrated in the next Section, in the analysis of experiments on second layer nucleation.

Eq. (16) assumes that atoms landing on the terrace because they are steered behave as unsteered ones. This is not exact, because the extra flux $F d \mathcal{P}$ is not deposited 


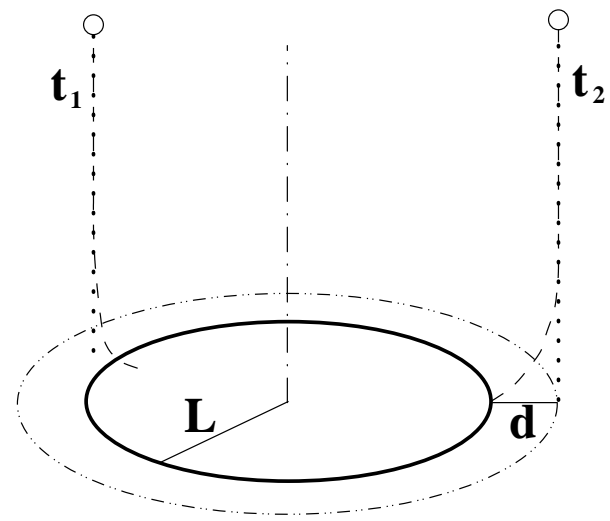

FIG. 3: Steering effects for normal deposition. Dotted and dashed lines represent the trajectories without and with steering, respectively. The adatoms following the trajectories $t_{1}$ and $t_{2}$ reach the terrace only because of the adatom-terrace interaction. $\mathcal{A}_{\text {eff }}$ corresponds to the area inside the dasheddouble dotted line.

uniformly, but close to the border of the terrace. Consequently, the probability $W$ is smaller than the value given by Eq. (5) and its use leads to an overestimate of steering effects. However, this is a second order effect that should be taken into account only if a more rigorous theory for deriving the incoming flux $\mathcal{F}$ were employed.

\section{SECOND LAYER NUCLEATION EXPERIMENTS}

The nucleation rate $\omega$, as we have discussed in Sec. II, is the rate of dimer formation on top of a terrace of fixed size $L$. Its direct experimental determination is very hard because it would require large statistics in single island measurements with a fixed size $L$.

Let us instead describe how 'second layer nucleation' experiments take place. A first possibility (see Sec. $\mathrm{VA}$ ) is to deposit a fraction of a monolayer on the substrate and to analyze the statistics of islands with a second nucleus on top. A second possibility (see Sec. VB) consists in preparing an ensemble of one monolayer high islands which is as uniform as possible, that is with the smallest dispersion in size and island-island distance. Afterwards, a new fraction of a monolayer is deposited and the statistics of islands with a second nucleus on top of them is studied.

The relevant quantity is clearly the probability $p(\tau)$ that a nucleation event has occurred on top of an island during a time $\tau$. For an ensemble of equivalent islands, this probability corresponds to the fraction $f(\tau)$ of islands with a second nucleus:20

$$
p(\tau)=f(\tau)=1-\exp \left[-\int_{0}^{\tau} d t \omega(L(t))\right]
$$

This expression clearly shows that comparison with a sec- ond layer nucleation experiment requires two separate pieces of information: the nucleation rate $\omega(L)$ and the growth law $L(t)$ of the islands. The former has a very general validity, because it does not depend on the details of the experiment. It does not even change if nucleation occurs on top of a mound: $\omega(L)$ is a 'single-island' problem and it is not affected by the growth dynamics of the overall surface. The latter piece of information, the growth law $L(t)$, is system dependent and an exact calculation of it is generally impossible: its determination involves, in principle, all the surface, in much the same way as the problem of submonolayer nucleation does.

Let us now determine in an approximate way $f(\tau)$, for a uniform array of islands of density $N_{\text {is }}\left(N_{\text {is }}\right.$ being the number of islands per lattice site). The growth law $L(t)$ is found according to a simple deterministic model 18,20 each island collects the atoms falling in the capture area $1 / N_{\text {is. }}$. If $\mathcal{A}_{i}=\gamma L_{i}^{2}$ is the initial area of the terrace, after a time $t$ :

$$
\mathcal{A}(t)=\mathcal{A}_{i}+\frac{F t}{N_{\text {is }}}, L(t)=\sqrt{L_{i}^{2}+\frac{F t}{\gamma N_{\text {is }}}} .
$$

Hence, if islands grow from the initial size $L_{i}$ to the final size $L_{f}$, the fraction of them with a second nucleus on top is

$$
f=1-\exp \left[-\frac{2 \gamma N_{\mathrm{is}}}{F} \int_{L_{i}}^{L_{f}} d L L \omega(L)\right] .
$$

The integral $\int d L L \omega(L)$, appearing in Eq. (19), can not be evaluated analytically for generic ES barriers: the reason is the complex $L$-dependence of $W$ [see Eq. (5)]. It is easily evaluated numerically.

It is useful to define a critical size $L_{c}$ : it is the (final) size of the islands, corresponding to a value $f=\frac{1}{2}$. In other words, an island grown up to the size $L_{c}$ has equal chance of having or not having a second nucleus on top. $L_{c}$ is determined by the implicit equation:

$$
\int_{L_{i}}^{L_{c}} d L L \omega(L)=\frac{\ln 2}{2} \frac{F}{\gamma N_{\mathrm{is}}} .
$$

We now reanalyze in detail two second layer nucleation experiments.

\section{A. $\mathbf{P t} / \mathbf{P t}(111)$}

This experiment is described in Ref. 13 and it has already been discussed, according to Eq. (8), in Ref. 18 . Using that expression for $\omega$, valid for strong barriers, the integral $\int d L L \omega(L)$ can be easily evaluated analytically. Here we do not assume that barriers are strong, we use the general expression (1), which will be later shown to be equivalent to (7), because of the validity of the relation $\tau_{\text {res }} \ll \tau_{\text {dep }}$.

In the experiment, $L_{i}=0$ and $L_{c}$ has been evaluated as follows: after deposition of a dose of platinum, 
TABLE II: The critical perimeter $\mathcal{P}_{c}$ and the density $N_{\text {is }}$ of islands, for the five different concentrations of CO.

\begin{tabular}{cccccc}
\hline \hline$p_{\mathrm{CO}}\left(10^{-11}\right.$ mbar $)$ & 0.5 & 10 & 47 & 95 & 190 \\
\hline $\mathcal{P}_{c}$ & 816 & 623 & 449 & 341 & 324 \\
$N_{\text {is }} \times 10^{5}$ & 2.75 & 2.1 & 1.83 & 1.76 & 1.65 \\
\hline \hline
\end{tabular}

the size $L_{-}$of the smallest island with a second nucleus on top, and the size $L_{+}$of the largest island without a second nucleus on top, have been determined experimentally for several STM topographs. The critical size has been approximated by the mean of their average values: $L_{c}=\frac{1}{2}\left(\left\langle L_{-}\right\rangle+\left\langle L_{+}\right\rangle\right)$.21

Experiments have been performed 13 at different $\mathrm{CO}$ partial pressures, $p_{\mathrm{CO}}$. In Table II we report $N_{\text {is }}$ and $\mathcal{P}_{c}$ for the different values of $p_{\mathrm{CO}} \cdot \mathcal{P}_{c}$ is the critical perimeter and it is three (six) times the critical size $L_{c}$, for triangular (hexagonal) islands. The values of the other physical parameters entering in Eqs. (5.,19,20) are: $F=5 \times 10^{-3} \mathrm{ML} / \mathrm{s}$, and $\nu=\nu_{0} \exp \left(-E_{d} / k_{\mathrm{B}} T\right)$, with $\nu_{0}=5 \times 10^{12} \mathrm{~s}^{-1}, E_{d}=0.26 \mathrm{eV}, T=400 \mathrm{~K}=0.0345$ $\mathrm{eV} / k_{\mathrm{B}}$. It is worth remarking 13 that island coalescence has already started for the smallest value of $p_{\mathrm{CO}}$. So, in that case the ES barrier $\Delta E$ is overestimated.

We now have all the ingredients in order to apply Eq. (20) and recover the value of the interlayer hopping rate, $\nu^{\prime}$, the only unknown quantity. In the hypothesis of a uniform barrier along the step, $\nu^{\prime}=$ $\nu_{0}^{\prime} \exp \left[-\left(E_{d}+\Delta E\right) / k_{\mathrm{B}} T\right]$. Two different values will be considered for the prefactor, $\nu_{0}^{\prime}=\nu_{0}=5 \times 10^{12} \mathrm{~s}^{-1}$ and $\nu_{0}^{\prime}=2.2 \times 10^{12} \mathrm{~s}^{-1}$. The latter value has been obtained by Field Ion Microscopy studies.22 As a general rule, if $\Delta E$ is the ES barrier obtained for a prefactor $\nu_{0}^{\prime}=\nu_{0}$, for a generic value $\nu_{0}^{\prime}$ the ES barrier changes to a value

$$
(\Delta E)^{\prime}=\Delta E+k_{\mathrm{B}} T \ln \left(\nu_{0}^{\prime} / \nu_{0}\right) .
$$

For $T=400 \mathrm{~K}$, changing $\nu_{0}^{\prime}$ by a factor two modifies the ES barrier by $24 \mathrm{meV}$; changing $\nu_{0}^{\prime}$ by a factor ten changes $\Delta E$ by $80 \mathrm{meV}$.

In Fig. A we report the results for the EhrlichSchwoebel barrier obtained using the exact nucleation rate given in Eq. (1),$\nu_{0}^{\prime}$ being equal to the two values mentioned more above. An inspection to the original STM images (Fig. 1 of Ref. 1) shows that for the smallest and the two largest values of $p_{\mathrm{CO}}$, islands are triangular, whilst in the other two cases the shape is less precisely defined. Therefore we have considered both hexagonal and triangular terraces: the upper and lower values of the error bars correspond to such cases. As one can see from the figure, at this temperature the effect of the indetermination of the terrace shape is comparable to an indetermination of $\nu_{0}^{\prime}$ of a factor two.

In Fig. 5 we compare our results with those obtained using the strong barrier approximation, Eq. (8): the latter is applicable for $\Delta E \geq 0.2 \mathrm{eV}$; for smaller values it overestimates $\omega$ and therefore it underestimates the ES

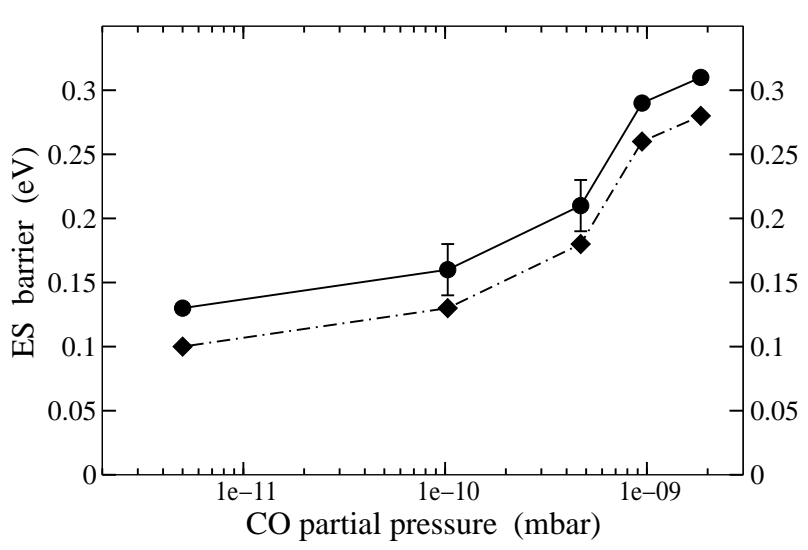

FIG. 4: Values of $\Delta E$, the ES barrier, for different $p_{\mathrm{CO}}$ values. Circles and diamonds refer, respectively, to $\nu_{0}^{\prime}=\nu_{0}=$ $5 \times 10^{12} \mathrm{~s}^{-1}$ and to $\nu_{0}^{\prime}=2.2 \times 10^{12} \mathrm{~s}^{-1}$. The error bars are due to the indetermination in the island shape.

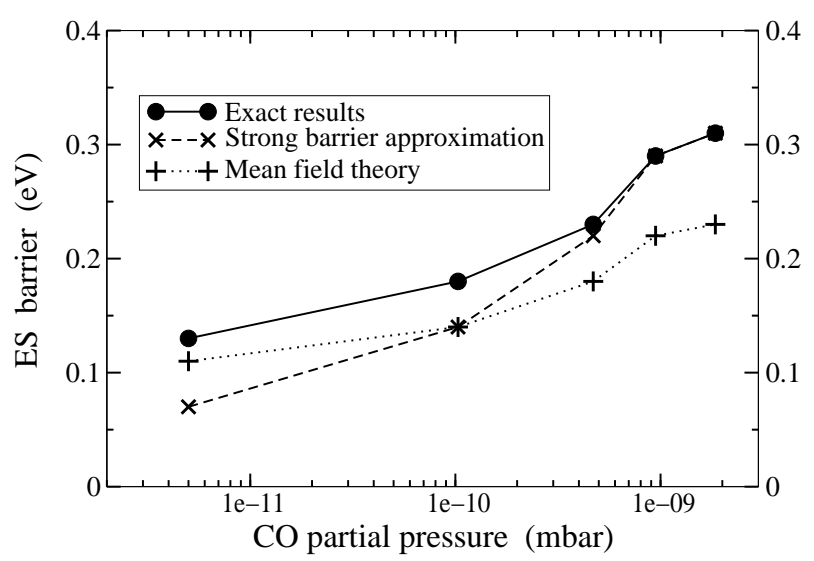

FIG. 5: Results with the exact nucleation rate (for $\nu_{0}^{\prime}=\nu_{0}$ and triangular islands) are compared to Mean Field Theory and to the values obtained from the 'strong barrier' approximation for $\omega$, see Eq. (8).

barrier. Mean Field Theory, also shown in the same figure, is generally incorrect.

We can now evaluate the ratio between the residence time and the deposition time. In the strong barrier limit, such ratio is

$$
\frac{\tau_{\text {res }}}{\tau_{\text {dep }}}=\alpha \gamma \frac{F}{\nu} \exp (\Delta E / T) L^{3} .
$$

For the largest barrier $(\Delta E=0.31 \mathrm{eV}), \tau_{\text {res }} / \tau_{\text {dep }}<$ 0.005. We conclude that for analysing the experiment on platinum, the use of formula (7) would be legitimate. Notice that, because of the exponential dependence of $\tau_{\text {res }}$ on $\Delta E$, larger values of the ES barrier quickly invalidate Eq. (7): $\tau_{\text {res }} \simeq \tau_{\text {dep }}$ for $\Delta E=0.4 \mathrm{eV}$.

Let us now consider the effect of steering on the value of the ES barrier. We repeat the evaluation of the ES barrier by considering an effective flux $\mathcal{F}=F(\mathcal{A}+\epsilon L)$ for several values of the parameter $\epsilon$, which measures the 'strength' of steering. Results are reported in Fig. 6 (note the log-linear scale). For triangular terraces on an 


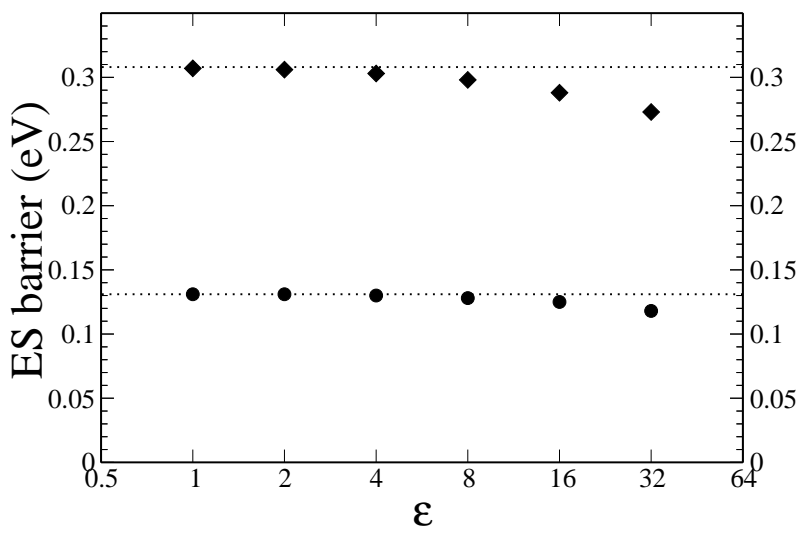

FIG. 6: The effect of steering on the interpretation of the second layer nucleation experiment on platinum, Ref. 13. Note the log-linear scale. Circles and diamonds refer, respectively, to $p_{\mathrm{CO}}=5 \times 10^{-12}$ mbar and $p_{\mathrm{CO}}=1.9 \times 10^{-9}$ mbar. Dotted lines mark the values of $\Delta E$ in the absence of steering.

fcc(111) surface, $\eta=3$, and $\epsilon$ is three times the (maximal) in-plane displacement of a particle landing close to the terrace edge. It is easy to check that, for the critical size of the terrace, the largest value of $\epsilon(\epsilon=32)$ corresponds to an incoming flux $\mathcal{F}$ increased by $60 \%$ for diamonds $\left(p_{\mathrm{CO}}=1.9 \times 10^{-9} \mathrm{mbar}\right)$ and by $24 \%$ for circles $\left(p_{\mathrm{CO}}=5 \times 10^{-12} \mathrm{mbar}\right)$. The ES barrier is seen to decrease for strong steering, because a larger effective flux requires a smaller barrier for producing the same nucleation rate. The fact that $\Delta E$ is less affected by steering when the barrier is smaller is intuitively clear: a smaller barrier implies a larger critical size $L_{c}$ and the larger is $L$, the smaller is the effect of steering, which is an edgeeffect.

\section{B. $\mathrm{Ag} / \operatorname{Ag}(111)$}

The experiment on silver reported in Ref. 4 is slightly different from the one on platinum. First, a quantity $\theta_{0}=$ $0.1 \mathrm{ML}$ is deposited. Afterwards an equal second dose $\Delta \theta=0.1 \mathrm{ML}$ is deposited at two different temperatures 23 $(T=120,130 \mathrm{~K})$ and the fraction $f$ of covered islands is determined as a function of the island radius $r$ (see Table III). The density of islands is determined by the relation $N_{\text {is }}=\theta_{0} / \mathcal{A}_{i}=\theta_{0} /\left(\gamma L_{i}^{2}\right)$, and $\Delta \theta=\theta_{0}$ implies $L_{f}=\sqrt{2} L_{i}$. Furthermore, $F=1.1 \times 10^{-3} \mathrm{ML} / \mathrm{s}, \nu_{0}=$ $2 \times 10^{11} \mathrm{~s}^{-1}$ and $E_{d}=0.097 \mathrm{eV}$.

Experimental data at different temperatures give the possibility - in principle - to determine both the barrier $\Delta E$ and the prefactor $\nu_{0}^{\prime}$ of the interlayer hopping rate. A large debate is going on in the literature about the interpretation of the experimental datal 12424 and the possibility that $\nu_{0}^{\prime} / \nu_{0} \gg 1$ (see below). Our contribution is to analyze the data with the correct theory and to ascertain the possible effect of nonuniform barriers and steering.

Inserting each pair of experimental values $(r, f)$ in Eq. (19) yields a value for the ES length, reported in
TABLE III: Experimental data for the fraction $f$ of covered islands as a function of the island radius $r$, and theoretical results for the corresponding ES lengths, $\ell_{i}=\ell_{\mathrm{ES}}\left(T_{i}\right)$.

\begin{tabular}{ccc|ccc}
\hline \hline \multicolumn{3}{c|}{$T_{1}=120 \mathrm{~K}$} & \multicolumn{3}{|c}{$T_{2}=130 \mathrm{~K}$} \\
$r(\AA)$ & $f$ & $\ell_{1}$ & $r(\AA)$ & $f$ & $\ell_{2}$ \\
7.0 & 0.0 & & 33.5 & 0.0345 & 5150 \\
11.5 & 0.02 & 300000 & 39.0 & 0.14 & 10200 \\
16.5 & 0.11 & 285000 & 47.0 & 0.47 & 17000 \\
21.5 & 0.24 & 180000 & 55.0 & 0.7 & 14800 \\
27.5 & 0.55 & 155000 & 63.0 & 0.85 & 11800 \\
32.0 & 0.88 & 200000 & 68.0 & 0.93 & 11400 \\
38.0 & 1.0 & & 77.0 & 1.0 & \\
\hline \hline
\end{tabular}

Table III, and consequently for the interlayer hopping rate, $\nu^{\prime}$, through the relation $\nu^{\prime}=\nu / \ell_{\mathrm{ES}}$.

For each temperature, we have used the value of $\ell_{\mathrm{ES}}$ obtained from the pair $(r, f)$ with $f$ as close as possible to $f=\frac{1}{2}$, i.e. with $r$ as close as possible to the critical size $L_{c}$, because it is the statistically most significant point: $f\left(L_{f}=27.5 \AA\right)=0.55$ at $T=T_{1}$, and $f\left(L_{f}=47 \AA\right)=0.47$ at $T=T_{2}$. Using the relations

$$
\frac{\Delta E}{k_{\mathrm{B}}}=\frac{T_{1} T_{2}}{\Delta T} \ln \left(\frac{\ell_{1}}{\ell_{2}}\right), \quad \frac{\nu_{0}^{\prime}}{\nu_{0}}=\frac{1}{\ell_{1}}\left(\frac{\ell_{1}}{\ell_{2}}\right)^{T_{2} / \Delta T},
$$

where $\Delta T=T_{2}-T_{1}$, we finally obtain $\Delta E=0.30 \mathrm{eV}$ and $\nu_{0}^{\prime} / \nu_{0}=1.9 \times 10^{7}$.

The value found for the prefactor $\nu_{0}^{\prime}=4 \times 10^{18} \mathrm{~s}^{-1}$, is huge and it is comparable to a previous analysis 18 of the same data using the approximate formula (8) for the nucleation rate.

A possible alternative way to interpret the data is to arbitrarily set $\nu_{0}^{\prime}=\nu_{0}$, and allow the ES barrier to depend on $T$. In this way we find $\Delta E \simeq 0.12 \mathrm{eV}$ at $T=120$ $\mathrm{K}$ and $\Delta E \simeq 0.11 \mathrm{eV}$ at $T=130 \mathrm{~K}$. Hence we find a significant difference for the barrier strength despite two rather close temperatures. This difference persists even if we suppose that $\nu_{0}^{\prime}$ is somewhat larger than $\nu_{0}$ (e.g., $\left.\nu_{0}^{\prime} / \nu_{0}=10^{2}\right)$. The values of $\Delta E$ change according to Eq. (21), but their difference

$$
\delta(\Delta E)^{\prime}=\delta(\Delta E)-k_{\mathrm{B}} \Delta T \ln \left(\nu_{0}^{\prime} / \nu_{0}\right)
$$

is almost unchanged, because $k_{\mathrm{B}} \Delta T \approx 10^{-3} \mathrm{eV}$ is smaller than $\delta(\Delta E) \equiv \Delta E(120 \mathrm{~K})-\Delta E(130 \mathrm{~K}) \approx 10^{-2} \mathrm{eV}$.

We can wonder whether experimental indeterminations can explain the large value of $\delta(\Delta E)$, or equivalently of $\nu_{0}^{\prime} / \nu_{0}$. As remarked in Ref. 因, island radii have been determined experimentally with a precision of about $5 \AA$. Taking into account the indetermination in $r$, the average values of $\ell_{1,2}$ are

$$
\ell_{1} \approx 180000 \pm 80000 \quad \ell_{2} \approx 14000 \pm 4000 .
$$

However, even if we consider the smallest $\ell_{1}$ and the largest $\ell_{2}$ [in order to minimize $\Delta E(120 \mathrm{~K})$ and to maximize $\Delta E(130 \mathrm{~K})$ ], we still have that $\delta(\Delta E)$ is of order $0.01 \mathrm{eV}$ [more precisely, $\delta(\Delta E)=8 \mathrm{meV}$ ]. 
Let us now discuss whether the unexpected experimental results can be the effect of non uniform barriers. Assuming that the prefactor $\nu_{0}^{\prime}$ is equal or even a bit larger than $\nu_{0}$, we have shown that $\delta(\Delta E) \approx 10 \mathrm{meV}$. If a scenario with two types of barriers applies, Eq. (14) gives the relation

$$
\delta(\Delta E) \approx k_{\mathrm{B}} \bar{T} \ln \left(\frac{c_{2}}{c_{1}}\right)
$$

between the difference in the ES barriers and the kink concentrations, $c_{1}$ and $c_{2}$. In the previous equation, $\bar{T}=$ $\frac{1}{2}\left(T_{1}+T_{2}\right)$ is the average temperature. A value $\delta(\Delta E) \approx$ $0.01 \mathrm{eV}$ would therefore require that the concentration $c_{2}$ of kinks at $T=130 \mathrm{~K}$ is two or three times larger than the concentration $c_{1}$ of kinks at $T=120 \mathrm{~K}$, which seems to be unlikely. 25

We finally consider the effect of steering. Increasing the value of the parameter $\epsilon$ reduces slightly the spreading of the ES lengths (third columns in Tab. III), but the value of $\delta(\Delta E)$ remains practically unaffected. Alternatively, if we consider $\nu_{0}^{\prime}$ as a free parameter, the ratio $\nu_{0}^{\prime} / \nu_{0}$ is smaller than without steering, but still too large: for $\epsilon=30$ we find $\Delta E=0.23 \mathrm{eV}$ and $\nu_{0}^{\prime} / \nu_{0}=1.5 \times 10^{5}$. We conclude that neither steering effects nor the presence of nonuniform barriers are enough to allow a reasonable interpretation of the experimental results of Ref. 4 .

\section{DISCUSSION AND CONCLUSIONS}

The present paper has three main goals. i) Provide a complete list of the correct formulas to be used for the interpretation of data from second layer nucleation experiments; ii) Extend the 'standard' model of irreversible nucleation and take into account the effect of steering and nonuniform ES barriers; iii) Apply the theoretical framework to reconsider some experimental results. Let us discuss these issues in detail

It is now well established 128 that Mean Field Theory 20 is not appropriate to study the problem of nucleation on top of a terrace and the reason of its failure has been clearly understood. 5 In Sec. II we give the most general formula for the nucleation rate, Eq. (1), and several approximate formulas which can be used in the relevant limits. With respect to our recent papers on the same problem, we are now able to provide a very good simple analytical expression for the probability $W$ that two atoms meet (Eq. 5), valid for any barrier strength. We also provide the numerical values for all parameters appearing in $W$ and in $\tau_{\text {res }}$, which depend on the shape of the terrace and on the symmetry of the underlying lattice, see Table 1 and App. B.

Mainly because of the complicated expression for $W$, it is not possible to integrate analytically the nucleation rate, Eqs. (19,20), and write explicitly, e.g., the critical size $L_{c}$ as a function of all the physical parameters, $F, T, \nu, \nu^{\prime}, \ldots$ This is not a real limitation, indeed, because performing such one dimensional numeri- cal integration is straightforward. In the limit of strong barriers $18 \omega \sim L^{5}$ and the analytical integration is possible. However, that approximation may be inaccurate, as we have shown in the case of platinum below $\Delta E=0.2$ $\mathrm{eV}$.

In Sec. III we have considered the possibility that the additional ES barrier at steps is not homogeneous. The case of a generic distribution of barriers can not be tackled analytically, but if barriers are everywhere large enough to keep the adatom density uniform, the problem is solvable. For a simple bimodal distribution (the barrier is equal to $\Delta E_{k}$ in special kink sites and equal to the larger value $\Delta E_{0}$ elsewhere) Eq. (14) indicates that the system behaves as it had a single effective barrier $\Delta E_{\text {eff }}$ equal to the kink barrier plus a correction depending on the kink concentration and temperature. This result shows that the same experimental system displays different effective barriers at different temperatures, or at different growth conditions.

In Sec. IV we have studied the effects of the 'incoming atom'-substrate interaction (steering) on the total flux landing on a top terrace. A detailed treatment would require to consider a realistic inter-atomic potential and to specify the energy and the inclination of the incoming particles. We have introduced a minimal model where the 'strength' of steering and all the above variables are included in a single adimensional parameter, $\epsilon=\eta d$. $\eta$ is a geometric factor and $d$ represents the (maximal) inplane deviation of an incoming atom.

Finally, in Sec. V we reconsider the data concerning two experiments of second layer nucleation, which had already been analyzed in Ref. 18, using the strong barrier approximation, Eq. (8).

In the case of $\mathrm{Pt} / \mathrm{Pt}(111)$, the additional barrier varies from a small value $(\Delta E \lesssim 0.1 \mathrm{eV})$ for the clean sample, to a value of order $0.3 \mathrm{eV}$ when terrace steps are fully decorated with CO. We stress that these values should be meant as effective ones. If steering plays a prominent role, the effective barrier decreases, because more adatoms are expected to land on the terrace. If the landing point of an adatom is displaced up to five interatomic distances, $d=5$, the largest barrier $\Delta E=0.31 \mathrm{eV}$ decreases by $20 \mathrm{meV}$; if $d=10$, the reduction is twice as large.

The case of $\mathrm{Ag} / \operatorname{Ag}(111)$ is very debated 824 and the question of the actual values of $\Delta E$ and $\nu^{\prime}$ is still open, requiring additional experiments at different temperatures. The interpretation of the data by Bromann et al. easily summed up. If the prefactor $\nu_{0}^{\prime}$ is assumed equal to $\nu_{0}$, the barrier is of order $0.12 \mathrm{eV}$ and it differs by about $10 \mathrm{meV}$ at the two temperatures, $T_{1}=120 \mathrm{~K}$ and $T_{2}=130 \mathrm{~K}$. This difference, $\delta(\Delta E)$, between the two barriers can be reduced if $\nu_{0}^{\prime}$ is allowed to increase. However, only an exponentially large value of $\nu_{0}^{\prime} / \nu_{0}$ [see Eq. (24)] would imply a noticeable reduction of $\delta(\Delta E)$. If $\nu_{0}^{\prime} / \nu_{0} \simeq 2 \times 10^{7}$, the two barriers are both equal to $0.30 \mathrm{eV}$. Data on the fraction of covered islands at different sizes give different barriers. The spreading of $\Delta E$ 
at a given temperature can be accounted for by the error bar on the determination of the radius $r$; however, this is not enough to explain the difference between $\Delta E\left(T_{1}\right)$ and $\Delta E\left(T_{2}\right)$. Steering effects are not able to make the difference consistent either. In principle, we expect two different effective barriers, at the two temperatures. Application of the formula (14) suggests that the value of $\delta(\Delta E)$ can be explained by a larger kink concentration at the highest temperature, $c_{k}\left(T_{2}\right) / c_{k}\left(T_{1}\right) \approx 2$, but it is difficult to find a physical motivation for such a large ratio. We conclude by remarking that a completely different measurement 27 of the interlayer rate in the same system, but at a higher temperature, $T=300 \mathrm{~K}$, gives a barrier $\Delta E=0.13 \mathrm{eV}$ and a prefactor $\nu_{0}^{\prime}$ of the same order of $\nu_{0}$ [see also the discussion in Ref. 24(b)].

We believe that possible further developments in the problem of nucleation should include the following points: a direct quantitative determination of the parameter $\epsilon$; an improvement of Eq. (14) to include the case of weak barriers; a better assessment of the growth law $L(t)$ for the terrace; additional quantitative and controlled second layer nucleation experiments at different temperatures and for other systems as well.

\section{APPENDIX A: INTRA AND INTER-LAYER HOPPING RATES}

The quantity $\nu$ is the total hopping rate on a flat surface. The jump rate in a given direction is $\nu_{d}=\nu / z$, where $z$ is the coordination number $(z=4,6$ for a square, triangular lattice respectively).

If an atom is on an edge site, its coordination number is reduced by $\Delta z$, the number of missing neighbors. The directed hopping rate towards a missing site (i.e. towards the lower terrace) is equal to $\nu_{d}^{\prime}$ and it may differ from the hopping rate, $\nu_{d}$, towards a site of the same terrace. If we define $\nu^{\prime}=z \nu_{d}^{\prime}$, the absence of additional step-edge barriers is equivalent to write $\nu^{\prime}=\nu$.

Within this formalism, the escape rate from the terrace for an atom on an edge site is equal to $\Delta z \nu_{d}^{\prime}=(\Delta z / z) \nu^{\prime}$. Other authors, see e.g. Ref. 8, prefer defining $\nu^{\prime}$ as the escape rate $\left(\nu^{\prime}=\Delta z \nu_{d}^{\prime}\right)$, but in that case the absence of additional ES barriers does not match the condition $\nu^{\prime}=\nu$.

\section{APPENDIX B: CALCULATION OF SOME PARAMETERS FOR A CIRCULAR TERRACE}

The derivation of $\gamma$ for a circular terrace is straightforward, because the number $\mathcal{A}$ of atoms contained in a circle of radius $L$ is equal to the area $\pi L^{2}$ divided by the area per atom, $A_{a}$. Since $A_{a}=1$ for a square lattice and $A_{a}=\sqrt{3} / 2$ for a triangular one, we have $\gamma=\pi$ in the former case, and $\gamma=2 \pi / \sqrt{3}$ in the latter case.

The derivation of $\eta$, that is, of the number $\mathcal{P}$ of edge sites, is less trivial. If we change $L$ by a quantity $d_{L}$, the total number of atoms changes by $d \mathcal{A}=2 \gamma d_{L} L$. If we set $d_{L}$ so that only edge sites are comprised in the circular ring, we have $\eta=2 \gamma d_{L}$. Let $\phi$ be the angle between the vector joining an edge site with the center of the circle, and a nearest neighbor bond. If $\phi=0$, $d_{L}=1$, whilst for a generic $\phi, d_{L}=\cos \phi$. The value of $d_{L}$ entering in the relation between $\eta$ and $\gamma$ is just the average over $\phi$ of $\cos \phi$. Because of the different lattice symmetries, the average is evaluated between zero and $\pi / 4$, for a square lattice, and between zero and $\pi / 6$, for a triangular lattice. We therefore obtain $d_{L}=2 \sqrt{2} / \pi$ in the former case, and $d_{L}=3 / \pi$ in the latter case. In conclusion, we obtain $\eta=4 \sqrt{2}$ for the (100) lattice and $\eta=4 \sqrt{3}$ for the (111) lattice.

The derivation of $\Delta z$, the (average) number of missing neighbours per edge site, is easily found from $\mathcal{P}$. Let us first determine the number $N_{n n}$ of nn bonds which are cut by a circle of radius $L$. It is elementary to write that $N_{n n}=8 L$ for a square lattice and $N_{n n}=8 \sqrt{3} L$ for a triangular lattice. The quantity $\Delta z$ is nothing but the ratio between $N_{n n}$ and $\mathcal{P}$, so that: $\Delta z=\sqrt{2}$ for the (100) lattice and $\Delta z=2$ for the (111) lattice.

\section{APPENDIX C: CALCULATION OF $N_{\text {dis }}$}

We want to evaluate analytically the average number of distinct sites $N_{\text {dis }}$ visited by an adatom during its random walk on the terrace. Let us imagine that we perform $N_{\text {run }}$ times the following procedure. We let a random walker start from a site on the terrace and follow its trajectory until it leaves the terrace. For each run $r$ and each terrace site $s$ we define the quantity $n(r, s)$ to be 1 if site $s$ has not been visited during run $r$ and 0 otherwise. The average probability that site $s$ is not visited is

$$
n(s)=\lim _{N_{\text {run }} \rightarrow \infty} \frac{1}{N_{\text {run }}} \sum_{r=1}^{N_{\text {run }}} n(r, s) .
$$

All runs can be grouped according to the number $F \geq 1$ of 'traversals' across the terrace, defined as follows. Once the atom has arrived on an edge site, it has the probability $p_{\text {out }}=\Delta z / z$ to attempt to move outside and $p_{i n}=1-\Delta z / z$ to move to another site of the terrace: in the former case, the atom has the probability $a=\ell_{\mathrm{ES}} /\left(1+\ell_{\mathrm{ES}}\right)$ to stay there and the probability $1-a=1 /\left(1+\ell_{\mathrm{ES}}\right)$ to leave the terrace. The first traversal starts when the atom is deposited on the terrace and terminates when the atom reaches an edge site and tries to leave the terrace. At this point the atom may leave the terrace, in which case it has performed one traversal only, or it may stay on the terrace and start a new traversal. According to the this definition, we can write

$$
n(s)=\lim _{N_{\text {run }} \rightarrow \infty} \frac{1}{N_{\text {run }}} \sum_{F=1}^{\infty} N_{\text {run }}^{F}\langle n(r, s)\rangle_{F},
$$


where $N_{\text {run }}^{F}$ is the total number of runs made up of $F$ traversals, and $\langle\cdots\rangle_{F}$ is the average value on those runs only.

The quantity $n(r, s)$ is different from zero if and only if the site $s$ has not been visited in any traversal. We can write explicitly

$$
n(r, s)=\prod_{k=1}^{F} n_{k}\left(r_{F}, s\right),
$$

where $n_{k}$ is the variable $n$ referred to the $k$-th traversal of the run labelled $r_{F}$. Inserting ( $\overline{\mathrm{C} 3}$ ) in Eq. (C2) we obtain

$$
n(s)=\sum_{F=1}^{\infty} \mu(F)\left\langle\prod_{k=1}^{F} n_{k}\left(r_{F}, s\right)\right\rangle,
$$

where $\mu(F)=N_{\text {run }}^{F} / N_{\text {run }}$ is the probability that a single run is made up of $F$ traversals.

If a run goes on for $F$ traversals, it means that the atom has done $F$ attempts to descend, failing the first $(F-1)$ times and succeeding the last one. Therefore,

$$
\mu(F)=a^{F-1}(1-a) .
$$

We now make the approximation that distinct traversals are independent:

$$
\left\langle\prod_{k=1}^{F} n_{k}\left(r_{F}, s\right)\right\rangle \simeq\left\langle n_{1}\left(r_{F}, s\right)\right\rangle \prod_{k=2}^{F}\left\langle n_{k}\left(r_{F}, s\right)\right\rangle .
$$

Using Eqs. (C5, C6 $)$ in ( $\mathrm{C} 4)$, we obtain

$$
n(s) \simeq \sum_{F=1}^{\infty}(1-a) a^{F-1}\left\langle n_{1}\left(r_{F}, s\right)\right\rangle \prod_{k=2}^{F}\left\langle n_{k}\left(r_{F}, s\right)\right\rangle .
$$

Let us remind the meaning of $\left\langle n_{k}\left(r_{F}, s\right)\right\rangle$. It is the probability that site $s$ is not visited during the $k$-th traversal of a run composed of $F$ traversals. This quantity does not depend on $F$. We make the approximation that it does not depend on $k$ either, if $k \geq 2$. In simple words, we just define $\left\langle n_{1}(s)\right\rangle$ for the first traversal and $\left\langle n_{\mathrm{BB}}(s)\right\rangle$ for all the subsequent traversals. The lowerscript BB means that the traversal starts from and arrives at the boundary of the terrace.

Within this approximation,

$$
\begin{aligned}
n(s) & \simeq\left\langle n_{1}(s)\right\rangle(1-a) \sum_{F=1}^{\infty} a^{F-1}\left\langle n_{\mathrm{BB}}(s)\right\rangle^{F-1} \\
& \simeq\left\langle n_{1}(s)\right\rangle \frac{(1-a)}{1-a\left\langle n_{\mathrm{BB}}(s)\right\rangle} \\
& \simeq \frac{\left\langle n_{1}(s)\right\rangle}{1+\ell_{\mathrm{ES}}\left(1-\left\langle n_{\mathrm{BB}}(s)\right\rangle\right)} .
\end{aligned}
$$

The last approximation is to neglect the $s$-dependence in $\left\langle n_{\mathrm{BB}}(s)\right\rangle$ and $\left\langle n_{1}(s)\right\rangle$. Using the simple relation, $N_{\mathrm{dis}}=$ $\sum_{s}(1-n(s))$, we obtain

$$
W=\frac{N_{\mathrm{dis}}\left(L, \ell_{\mathrm{ES}}\right)}{\mathcal{A}}=\frac{\ell_{\mathrm{ES}} N_{\mathrm{dis}}^{\mathrm{BB}}+N_{\mathrm{dis}}(L, 0)}{\ell_{\mathrm{ES}} N_{\mathrm{dis}}^{\mathrm{BB}}+\mathcal{A}}
$$

where $N_{\text {dis }}(L, 0)=\left(1-\left\langle n_{1}(s)\right\rangle\right) / \mathcal{A}$ and $N_{\text {dis }}^{\mathrm{BB}}(L)=(1-$ $\left.\left\langle n_{\mathrm{BB}}(s)\right\rangle\right) / \mathcal{A}$ is the number of distinct sites visited by a particle starting from the boundary and reaching it again. Inserting the expression for $N_{\mathrm{dis}}^{\mathrm{BB}}(L)=N_{1} L / \ln \left(L / L_{1}\right)$ we obtain the explicit form of $W$

$$
W=\frac{N_{1} \ell_{\mathrm{ES}}+\frac{N_{0} L \ln \left(L / L_{1}\right)}{\ln \left(L / L_{0}\right)}}{N_{1} \ell_{\mathrm{ES}}+\gamma L \ln \left(L / L_{1}\right)} .
$$

\section{ACKNOWLEDGMENTS}

We gratefully thank Thomas Michely and Harald Brune for providing the original data of the two experiments discussed in Sec. VA and Sec. VB. Thomas Michely and Joachim Krug are acknowledged for their critical reading of the manuscript. Special thanks go to Ruggero Vaia for having suggested how to determine $\eta$ and $\Delta z$ for a circular terrace. Part of this work was done during the International Seminar "Models of Epitaxial Crystal Growth" held at the Max Planck Institute for the Physics of Complex Systems, Dresden, in March 2002.
* Electronic address: politi@ifac.cnr.it

$\dagger$ Electronic address: castella@pil.phys.uniroma1.it

1 P. J. Feibelman and T. Michely, Surf. Sci. 492, L723 (2001); M. Kalff, P. Smilauer, G. Comsa and T. Michely, ibid. 426, L447 (1999).

2 C.-M. Zhang, M. C. Bartelt , J.-M. Wen , C. J. Jenks , J. W. Evans and P. A. Thiel, Surf. Sci. 406, 178 (1998); L. Bardotti, C. R. Stoldt, C. J. Jenks, M. C. Bartelt, J. W. Evans and P. A. Thiel, Phys. Rev. B 57, 12544 (1998).

3 J. A. Stroscio, D. T. Pierce and R. A. Dragoset, Phys. Rev. Lett. 70, 3615 (1993); J. A. Stroscio and D. T. Pierce,
Phys. Rev. B 49, 8522 (1994).

${ }^{4}$ K. Bormann, H. Brune, H. Röder and K. Kern, Phys. Rev. Lett. 75, 677 (1995).

5 P. Politi and C. Castellano, Phys. Rev. E 66, 031605 (2002); 66, 031606 (2002).

6 C. Castellano and P. Politi, Phys. Rev. Lett. 87, 056102 (2001).

7 A. Pimpinelli, J. Villain, Physics of Crystal Growth (Cambridge University Press, Cambridge, 1998).

$8 \mathrm{~T}$. Michely and J. Krug, Islands, Mounds and Atoms: Crystal Growth far from Equilibrium (Springer, Berlin, 
2003).

9 J. Jacobsen, K. W. Jacobsen, P. Stoltze, and J. K. Nørskov, Phys. Rev. Lett. 74, 2295 (1995).

10 N. J. Shevchik, J. Non-Cryst. Solids 12, 141 (1973).

11 S. van Dijken, L.C. Jorritsma and B. Poelsema, Phys. Rev. Lett. 82, 4038 (1999); Phys. Rev. B 61, 14047 (2000); B. Poelsema and S. van Dijken, in Atomistic aspects of epitaxial growth, edited by M. Kotrla, N. I. Papanicolau, D. D. Vvedenski, and L. T. Wille (Kluwer Academic Publishers, Dordrecht 2002), p. 165.

12 F. Montalenti and A. F. Voter, Phys. Rev. B 64, 081401 (2001).

13 M. Kalff, G. Comsa and T. Michely, Phys. Rev. Lett. 81, 1255 (1998).

14 When the ES length is large, we can neglect the term $(-1)$ appearing in its general definition.

15 With respect to our previous Refs. 5. 6, we have introduced the hopping rate $\nu$ rather than the diffusion constant $D=$ $\nu / 4$, in the expression (2) of the residence time. Therefore the new $\alpha$ and $\beta$ are four times larger than the old ones.

16 This is not true if the particle is initially confined to the edge of the terrace, but this case is not practically relevant.

17 B. D. Hughes, Random walks and random environments (Clarendon Press, Oxford, 1995).

18 J. Krug, P. Politi and T. Michely, Phys. Rev. B 61, 14037 (2000).
19 In Eq. (15) we are neglecting the existence of a kink between neighbouring straight steps.

20 J. Tersoff, A. W. Denier van der Gon and R. M. Tromp, Phys. Rev. Lett. 72, 266 (1994).

21 T. Michely (private communication).

${ }^{22}$ K. Kyuno and G. Ehrlich, Phys. Rev. Lett. 81, 5592 (1998).

23 Experimental data are available at $T=70 \mathrm{~K}$ as well, but they can not be used in the present context, because $f=1$ for all observed islands.

24 (a) K. R. Roos and M. C. Tringides, Phys. Rev. Lett. 85, 1480 (2000); 87, 149602 (2001); 87, 149604 (2001); 87, 149606 (2001); J. Krug, ibid. 87, 149601 (2001); K. Morgenstern and F. Besenbacher, ibid. 87, 149603 (2001); S. Heinrichs and P. Maass, ibid. 87, 149605 (2001); (b) Z. Chvoj and M. Tringides, Phys. Rev. B 66, 035419 (2002).

25 Because of kinetic effects the number of kinks should decrease with increasing $T$. Because of thermodynamical effects, it should increase, but $k_{\mathrm{B}} \Delta T \approx 1 \mathrm{meV}$ is much smaller than the kink energy.

26 J. Rottler and P. Maass, Phys. Rev. Lett. 83, 3490 (1999); S. Heinrichs, J. Rottler, and P. Maass, Phys. Rev. B 62, 8338 (2000).

27 K. Morgenstern, G. Rosenfeld, E. Lægsgaard, F. Besenbacher, and G. Comsa, Phys. Rev. Lett. 80, 556 (1998). 